\title{
Reducing neuroinflammation in Parkinson's disease with dietary compounds
}

\author{
Steve Michael Blake*, Panida Piboolnurak, Patricia Lynn Borman, Thomas Harding and Catherine Peterson \\ Blake
}

Maui Memory Clinic, Novant Health Neurology, and University of Hawaii, Department of Geriatrics, NC and HI, USA

GSC Biological and Pharmaceutical Sciences, 2022, 18(02), 026-037

Publication history: Received on 28 December 2021; revised on 31 January 2022; accepted on 02 February 2022

Article DOI: https://doi.org/10.30574/gscbps.2022.18.2.0052

\begin{abstract}
Emerging evidence indicates that chronic brain inflammation may lead to oxidation and death of dopaminergic neurons in Parkinson's disease (PD). Increased levels of pro-inflammatory cytokines have been found in brain and cerebrospinal fluid in PD patients. Higher inflammation is associated with poorer motor function and cognition in PD. Data from postmortem studies show the increased presence of activated microglial cells expressing inflammatory cytokines in the substantia nigra of PD patients. When aberrant proteins, such as alpha-synuclein, activate microglia, a self-sustaining cycle of neuroinflammation may kill off dopaminergic brain cells. Reducing brain inflammation may slow down neuronal death and disease progression, especially if instituted early in disease progression. We will review dietary triggers of inflammation and ways to ameliorate chronic inflammation. We will introduce four key dietary compounds that can be avoided to decrease inflammation and oxidation: Arachidonic acid, lipopolysaccharides, dairy products, and advanced glycation endproducts. We will also review many dietary compounds that can reduce chronic inflammation and oxidation, potentially reducing the risk and progression of PD.
\end{abstract}

Keywords: Microglia; Alpha-synuclein; Parkinson's disease; Inflammation; Dietary; Cytokine

\section{Introduction}

Parkinson's disease (PD) is characterized by bradykinesia, tremor, and rigidity resulting from the degeneration and cell death of dopaminergic neurons in the nigro-striatal pathway. This leads to progressive malfunction of the nigro-striatal system and disease progression. Neuronal cell bodies, located in the compact part of the substantia nigra, send long axons into the striatum. These projections are vital for motor functions and they are particularly vulnerable to degeneration in PD. The pathological signs of PD are loss of dopaminergic neurons and accumulation of misfolded alphasynuclein in degenerating neurons in the substantia nigra pars compacta.

Parkinson's disease starts long before diagnosis. By the time of diagnosis, postmortem analyses have shown that about $30 \%$ of dopaminergic neuronal cell bodies in the substantia nigra and $50 \%$ of dopamine axon terminals in the dorsal putamen are lost [1]. The available medical therapies only treat the symptoms of the disease without stopping or slowing the neurodegenerative process [2,3]. The reasons why dopaminergic neurons die in PD are not fully known. Emerging evidence indicates a role for brain inflammation and resulting oxidation in this loss of dopaminergic neurons. Movement areas of the brain are susceptible to inflammation and oxidation. Excess, chronic inflammation may increase oxidative damage to dopaminergic neurons. Reducing brain inflammation may slow down neuronal death and disease progression, especially if instituted early in disease progression. Some food substances increase inflammation, while others reduce inflammation, potentially slowing neurodegeneration in PD.

\footnotetext{
*Corresponding author: Steve Michael Blake, Email:steve@drsteveblake.com Phone: (808) 280-6894

Maui Memory Clinic, Director of Nutritional Neuroscience, Wailuku, HI, USA. HI, USA.

Copyright ( 12022 Author(s) retain the copyright of this article. This article is published under the terms of the Creative Commons Attribution Liscense 


\section{Inflammation}

Increased levels of pro-inflammatory cytokines have been found in the PD patient brain and cerebrospinal fluid (CSF). PD patients also display signatures of global inflammation in the blood, gut, and central nervous system (CNS) [4]. There is a pattern of systemic inflammatory markers in patients with PD that correlates with the clinical stage of the disease, indicating the contribution of peripheral inflammation in PD progression [5]. This includes elevated blood and CSF cytokine levels, activated microglia, infiltration of peripheral immune cells into the CNS, hyper-reactive circulating immune cells, and a dysregulated gut microbiome [6].

Studies that have looked at CSF in the early stages of PD (disease duration 3 years or less) have found increased levels of interleukin-1-beta (IL-1ß) and interleukin-6 (IL-6) compared to control subjects. These cytokines were not found in the blood in these early stages, indicating that the cytokines have an origin in the brain. Postmortem studies have detected increased brain tissue levels of tumor necrosis factor-alpha (TNF- $\alpha$ ), IL-1 $\beta$, IL-6, interferon-gamma (IFN- $\gamma$ ), and transforming growth factor-beta (TGF- $\beta$ ) in the PD striatum compared to controls $[7,8]$.

\subsection{Higher inflammation is associated with poor motor function and cognition in PD}

Patients with PD who have a high inflammation score have significantly more severe motor impairment and reduced performance. Higher inflammation is associated with impaired activities of daily living. Reducing inflammation lowered the risk of declining activities of daily living by 54\% [9]. Higher serum levels of TNF- $\alpha$ correlate with faster decline of motor function over 3 years, and higher IL-1 $\beta$ and interleukin-2 (IL-2) correlate with faster cognitive decline [10].

\subsection{Excess cytokines kill off dopaminergic neurons}

Inflammatory cytokines like TNF- $\alpha$ and IL-1 $\beta$ can kill off dopaminergic neurons by damaging mitochondria and inducing cell death. Post-mortem analyses indicate that cytokines, including TNF- $\alpha$ and IL-1 $\beta$, are significantly elevated in the area of the substantia nigra where maximal destruction of vulnerable dopaminergic neurons occurs in PD patients [11].

\subsection{The inflammatory cytokine tumor necrosis factor-alpha increases dopaminergic neuron death}

The inflammatory cytokine TNF- $\alpha$ is found in much higher levels in Parkinson's brains. TNF- $\alpha$ can activate abundant numbers of microglia in the midbrain, increasing inflammatory responses that lead to damaging reactive oxygen and nitrogen radicals. TNF- $\alpha$-dependent microglial activation in the substantia nigra increases oxidative stress that damages dopaminergic neurons. TNF- $\alpha$ in the substantia nigra and striatum increases neuronal death [12]. TNF- $\alpha$ is a critical player in pathways that kill dopaminergic neurons [13].

\subsection{Inflammation and cytokines}

Data from postmortem studies showed the increased presence of activated microglial cells expressing TNF- $\alpha$, IL- $1 \beta$, IL6 , and IFN- $\gamma$ in the substantia nigra of PD patients. Higher amounts of T-cells were also found. Many of these inflammatory cytokines were also found to be increased in cerebrospinal fluid and blood plasma of PD patients. Imaging studies of microglial activation in PD patients showed increased inflammation levels in the pons, striatum, and frontal, temporal, and occipital cortical regions, compared to age-matched healthy controls. Increased microglial activation correlated with worse performance in general cognitive testing [14].

\subsection{Blood-brain barrier and inflammation}

Increased levels of peripheral cytokines can act on the endothelial cells of the blood-brain barrier (BBB), potentially inducing an increase in vascular permeability [15]. Vascular abnormalities in PD patients associated with neuroinflammation can increase BBB leakage in the striatum and in the midbrain [16]. Neuroinflammation may be triggered by infections, brain trauma, stroke, rheumatoid arthritis, atherosclerosis, Crohn's disease, multiple sclerosis, or environmental toxins like pesticides. Neuroinflammation can increase the permeability of the BBB to allow entry of lymphocytes and macrophages into the brain, increasing inflammation [17].

\subsection{Evidence for anti-inflammatory drug use in reducing risk of Parkinson's disease}

A large prospective study of hospital workers indicated that the incidence of PD in chronic users of over-the-counter non-steroidal anti-inflammatory drugs (NSAIDs) was $46 \%$ lower than that of age-matched non-users. NSAIDs reduce inflammation by inhibiting cyclooxygenase (COX) activity [18]. More recent studies are showing a modest or no effect of NSAIDs on PD risk [19]. 


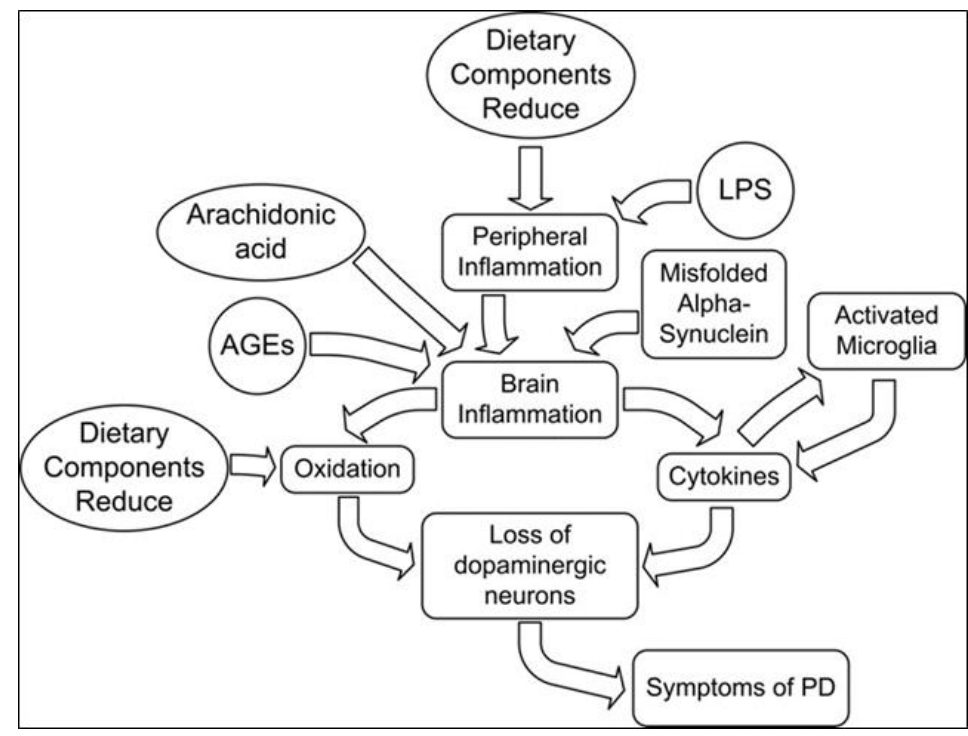

Figure 1 Inflammation and Parkinson's disease (PD). AGE = advanced glycation endproducts LPS = lipopolysaccharides

\section{Microglia}

There is good evidence from human PD studies that activated microglia are increased early in the disease process, certainly within 3 years from diagnosis.

\subsection{Resting and activated microglia}

In normal physiological conditions, microglia are in a resting and surveillance mode. Resting or mildly activated microglia secrete neurotrophic factors, remove toxic substances, and participate in neuronal repair, remodeling, and synaptic pruning [20]. Microglia, when moderately activated, can scavenge excess neurotoxins and remove dying cells and cellular debris. Moderately activated microglia can repair axons of dopamine neurons by releasing brain-derived neurotrophic factor (BDNF) [21].

\subsection{Triggering of microglia}

If microglia are primed by a stimulus, like lipopolysaccharide (LPS) or IFN- $\gamma$, this exposure can trigger death of dopaminergic neurons. This can shift microglial activities from neuroprotective to neurotoxic. Instead of protecting neurons, microglia can overproduce reactive oxygen species, leukotrienes, and cytokines [22].

\subsection{Activated microglia secrete pro-inflammatory cytokines}

As a part of the innate immune system, microglia are able to sense neuron-derived danger-associated molecular patterns, resulting in microglial activation. Activated microglia secrete pro-inflammatory cytokines such as IL-1 $\beta$, IL-6, IFN- $\gamma$, and TNF- $\alpha$. Other common stimuli for microglial activation, both in vitro and in vivo, are beta-amyloid and alphasynuclein [23]. Microglial activation is among the initial inflammatory events that takes place in PD. Any treatment that inhibits microglial activation may be most effective at an early PD stage to slow down or stop further neurodegeneration [24].

\subsection{Microglia and PD progression}

Patients with early PD have markedly elevated neuroinflammation in the pons, basal ganglia, striatum, and frontal and temporal cortical regions compared to age-matched controls. This indicates that increased microglial activation in early PD is likely to occur in parallel with loss of dopaminergic neurons. Reducing inflammation may reduce progression [25].

\subsection{Astrocytes and oligodendrocytes}

The brain's immune system also includes astrocytes that regulate the environment between cells, partially by adjusting glutamate, a stimulating neurotransmitter. Astrocytes may become activated by chemicals, physical damage, or 
ischemia. Also, activated oligodendrocytes can release inflammatory cytokines and inhibit regeneration of axons [26, 27].

\section{Oxidative stress}

\subsection{How brain inflammation creates damaging oxidation}

Chronic brain inflammation can increase the susceptibility of neurons to injury by inducing oxidative stress. Activated microglia and astrocytes can produce high levels of reactive oxygen species when triggered by inflammation. Neuroinflammation increases oxidative stress. Long before clinical symptoms of PD develop, oxidative stress can kill off dopaminergic neurons in the substantia nigra in the brain. The progression of PD involves further cell death with excess oxidation as a contributor [28].

\subsection{Dopaminergic brain cells are vulnerable to oxygen damage}

The substantia nigra is especially vulnerable to oxidation and resulting cell death. The brain's high energy use contributes to mitochondrial production of free radicals. The substantia nigra is lower in protective glutathione than other brain areas. In addition, metabolism of dopamine can result in excess hydrogen peroxide if antioxidants are insufficient [29]. Hydrogen peroxide is a damaging free radical that can be neutralized by glutathione peroxidase or catalase.

\subsection{More oxygen damage is found in dopaminergic brain cells in PD}

Protein oxidation in the substantia nigra of those with PD is double that found in those without this neurodegenerative disorder. Oxidative damage to the DNA of dopaminergic cells has been found to be 16 times greater in Parkinson brains compared to other brains. This can lead to cell death through damaged mitochondria and apoptosis [30]. The neuronal vulnerability to oxidative stress is a key aspect of neurodegenerative processes. Oxidative stress from cerebral hypoxia and reperfusion may also be a risk factor for neurodegeneration [31].

\subsection{Atherosclerosis can increase Parkinson's disease risk}

Atherosclerotic plaque deposits in aging individuals can disrupt the function of the brain through a process of chronic inflammation that results from interactions between oxidized low-density lipoproteins, immune cells, and endothelial cells. As such, atherosclerosis may be an age-associated disease that increases risk or accelerates development of sporadic Parkinson's disease by promoting neuroinflammation-dependent oxidative stress in the brain [32].

\subsection{Glutathione protects brain cells, but is lower in PD}

Excess inflammation can increase the expression of cytokines that promote damaging oxygen radicals. Glutathione protects mitochondria and dopaminergic neurons from oxidation, but reduced levels of glutathione are found in the substantia nigra of PD patients compared to age-matched controls [33]. Adequate selenium is necessary for glutathione to function and protect dopaminergic neurons.

\section{Protein mis-folding and alpha-synuclein}

\subsection{Protein mis-folding can promote inflammation and cell death}

Protein mis-folding and clumping may contribute, over time, to the death of neurons by stimulating inflammation and oxidation. An excess of alpha-synuclein ( $\alpha$-SYN) protein can increase dopaminergic neuron loss [34]. Alpha-synuclein aggregates can trigger a cascade of pro-inflammatory mediators that include TNF- $\alpha$, IL-1 $\beta$, IL- 6 , COX-2, and inducible nitric oxide synthase. Alpha-synuclein can trigger the active state of microglia [35]. When aberrant proteins, such as $\alpha$ SYN, activate microglia, a self-sustaining cycle of neuroinflammation may kill off dopaminergic brain cells. This can occur in PD with $\alpha$-SYN aggregates [36].

In PD patients, $\alpha$-SYN can be released from damaged neurons into the extracellular fluid. Here it activates microglia and induces a pro-inflammatory response with production and secretion of IFN- $\gamma$ in addition to IL-1 $\beta$, IL-6, and TNF- $\alpha$. When $\alpha$-SYN is assembled into oligomers and fibrils, it is thought to be toxic to neurons, leading to neurodegeneration [37]. 
Alpha-synuclein inclusions alone cannot be blamed for PD. There are cases of healthy people with high Lewy body load, but without symptoms. Lewy body load does not necessarily correlate with the severity of symptoms, suggesting that there may be other mechanisms at work that explain the pattern of motor and non-motor symptoms in patients [38].

\subsection{Environmental toxins can increase alpha-synuclein in Lewy bodies}

Chemical products used in herbicides and pesticides, including paraquat and rotenone, have been shown to inhibit mitochondrial complex I and promote $\alpha$-SYN aggregation. Environmental toxins can increase $\alpha$-SYN mis-folding and deposition into Lewy bodies [39].

\section{Dietary factors that increase chronic inflammation}

\subsection{How dietary arachidonic acid creates damaging inflammation}

Dietary arachidonic acid comes only from animal fat. Higher arachidonic acid intake increases the risk of PD [40]. Arachidonic acid is processed by lipoxygenase enzymes to become hydroperoxyeicosatetraenoic acid and then the 4series of inflammatory leukotrienes. Leukotrienes made from arachidonic acid increase inflammation and programmed cell death in the mid brain. Arachidonic acid also increases the oxidation of dopamine. Prostaglandins from arachidonic acid (PGE2 and PGJ2) have a toxic effect on dopaminergic neurons [41].

\subsection{Lipopolysaccharides may trigger Parkinson's disease}

When meat, poultry, or milk is cooked, bacteria are killed. The left-over membranes of gram-negative bacteria, known as Lipopolysaccharide (LPS), or endotoxin, have been implicated to play a role in progressive neurodegeneration in PD [42]. LPS can enter the bloodstream of people who eat meat, chicken, or cheese, assisted by the excess saturated fatty acids in these foods $[43,44,45]$. LPS can also be derived from dysbiosis in the gut or from infections. LPS, together with saturated fatty acids, can be packaged into chylomicrons in the enterocytes and enter the bloodstream [46]. LPS increases oxidative stress by increasing inflammation. There is evidence of a direct role of peripheral LPS on the central nervous system, including microglia. LPS may trigger inflammation in the brain via cluster of differentiation 14 (CD14) LPS receptors [47]. LPS can degrade the neurons in the substantia nigra and striatum and may be an important trigger for Parkinson's disease [48].

LPS was shown to trigger an inflammatory reaction that activates microglia and induces selective and irreversible damage to dopaminergic neurons while sparing serotonergic neurons [49]. Bacterial LPS resulted in a robust cytokine response in both PD and controls [50]. LPS has been shown to increase inflammation in the dopaminergic neurons of the substantia nigra, contributing to pathogenesis of PD. LPS stimulates astrocytes and microglia in the central nervous system to secrete inflammatory cytokines such as TNF- $\alpha$, IL-6, and IFN- $\gamma$. Microglial activation via LPS preceded the neuronal degeneration [51].

\subsection{Association of milk and cheese, oxidative damage, and risk of PD}

The risk of PD increases with increased milk and cheese consumption [52]. Parkinson's disease risk raises $40 \%$ in those who consume dairy products. Those who consumed more than 2.9 servings of dairy products (compared with 1 serving) per day, had an $80 \%$ increased risk of PD [53]. Four cups of milk daily was found to triple the risk of PD [54].

Dairy products may decrease serum uric acid levels, thus reducing an important antioxidant. Conversely, high serum urate levels have been associated with a decreased risk of PD and a lower rate of disease progression. Uric acid is commonly found in asparagus, cauliflower, spinach, lentils, and other legumes. Dairy foods also increase exposure to pesticides, especially lipophilic organochlorines and polychlorinated biphenyls that have been linked to higher PD risk [55]. Dairy products increased the risk of Parkinson's disease 2.3 times and neurotoxin contaminants are suspected [56].

\subsection{Advanced glycation endproducts increase inflammation in the brain}

Advanced glycation endproducts (AGEs) are a group of compounds formed by the Maillard chemical process of nonenzymatic glycation of free amino groups of proteins, lipids, and nucleic acids. AGEs are formed when meat, chicken, or fish is prepared by high-temperature processing, such as broiling, barbequing, or deep-frying. AGEs are also found in aged cheese. The presence of water in plant foods prevents the formation of AGEs. About $10 \%$ of AGEs are absorbed into circulation from the gut (the smaller fragments) [57]. 
AGEs, by triggering the brain receptor for advanced glycation endproducts (RAGE), enhance oxidative stress, innate immune activation, protein aggregation, and neuronal death in PD. Increased RAGE activation by AGEs was also correlated with a higher risk for PD development. When compared to healthy controls, PD patients have recently been shown to possess higher concentrations of AGEs in the substantia nigra and CSF [58].

RAGE triggering can increase neuroinflammation in PD by increasing nuclear factor kappa-b (NF- $k b$ ). Sesamin (from sesame seeds) and the standardized extract of ginkgo biloba may be promising phytochemical compounds that may delay microglial inflammation triggered by AGEs [59].

\section{Dietary compounds reducing inflammation}

Diets rich in unprocessed plant foods may reduce chronic inflammation and positively influence PD symptoms and progression of the disease [60].

\subsection{Short-chain fatty acids reduce inflammation}

Short-chain fatty acids are formed from plant fiber and resistant starch by the action of colonic bacteria [61]. Diets rich in plant fiber and resistant starch contribute to production of more short-chain fatty acids (SCFAs) in the large intestine, decreasing inflammation. Lower levels of these SCFAs are found in PD patients. Lower levels of SCFAs and higher levels of plasma cytokines are related to PD onset and symptom severity [62]. Vegan diets and the Mediterranean diet produce high quantities of anti-inflammatory SCFAs from plant fiber [63]. Probiotics and prebiotics can regulate defensin and promote mucin production, thus enhancing the protective barrier mechanism in the intestine [64].

\subsection{Curcumin from turmeric reduces inflammation and may be neuroprotective in PD}

Curcumin is a curcuminoid found in turmeric that scavenges free radicals and protects mitochondria by increasing glutathione. Curcumin can cross the BBB and is thought to be neuroprotective in PD. Curcumin can restore mitochondrial function, increase striatal dopamine, inhibit inflammatory markers, and reduce reactive oxygen species [65]. Curcumin reduced glial activation (microglia and astrocytes) and the transcription factor NF-kb [66].

\subsection{DHA and EPA reduce PD risk and reduce inflammation}

Large prospective cohort studies showed that polyunsaturated fatty acid intake was associated with reduced PD risk [67]. Eicosapentaenoic acid (EPA) and docosahexaenoic acid (DHA) inhibit IL-1 $\beta$-induced NF- $\kappa b$ activation and TNF- $\alpha$ to reduce inflammation in the gut, brain, and bloodstream [68].

\subsection{Resveratrol protects mitochondria and reduces inflammation}

Resveratrol, a polyphenol and a phytoalexin found in grapes, peanuts, berries, and pine bark, can inhibit the activation of microglia and subsequent inflammation. The anti-inflammatory action of resveratrol is attributed to the reduction of the inflammatory NF-kb signaling pathway in microglia. This inhibits nicotinamide adenine dinucleotide phosphate oxidase with a subsequent reduction of reactive oxygen species [69].

\subsection{Genistein from soy products reduces inflammation}

Genistein is found in soy beans, tofu, soy milk, and edamame. Genistein reduces inflammation by suppressing LPSinduced TNF- $\alpha$ and IL-6. It also has been found to block secretion of TNF- $\alpha$ and interleukin-1. Genistein treatment also downregulates cyclooxygenase-2 to reduce inflammatory eicosanoids [70]. Genistein has also been reported to have anti-apoptotic effects, reducing cell death in dopaminergic neurons [71].

\subsection{Antioxidant rich foods}

Improving diet to include more antioxidant-rich foods can reduce oxidative stress in PD. Reduction of oxidative stress can slow the progression of PD. Several dietary substances promote neuroprotective antioxidant action, including vitamin C, vitamin E, carotenoids, selenium, and polyphenols [72].

There is evidence that a higher intake of fruits and vegetables containing flavonoids is associated with a decreased risk of PD. Compared to a diet with a high intake of red meats, refined grains, refined sugars, and high-fat foods, a diet rich in fruits, vegetables, and whole grains reduced the risk of PD [73]. 


\subsection{Coenzyme $\mathbf{Q 1 0}$ is antioxidant and helps energy production in mitochondria}

Clinical trials involving Coenzyme Q10 have been conflicting. While one study reported a dose-dependent reduction in functional decline, another study observed only mild symptomatic benefit in PD [74].

\subsection{Sulforaphane protects dopaminergic neurons}

Sulforaphane is found in cruciferous vegetables such as broccoli, kale, and cabbage. Sulforaphane crosses the bloodbrain barrier and has been found in the ventral midbrain and striatum after ingestion. It increases intracellular glutathione levels, thus reducing oxidative stress. It also protects dopaminergic neurons from cytotoxicity by reducing toxic metabolites of dopamine breakdown [75]. Auto-oxidation of dopamine can result in damaging quinones and powerful oxidants, such as superoxide. Quinones can increase mitochondrial dysfunction and increase levels of $\alpha$-SYN. Sulforaphane was able to significantly reduce toxic quinone levels in dopaminergic neurons. Sulforaphane increases endogenous glutathione to normalize intracellular redox status. Also, sulforaphane induced a nuclear factor erythroid 2-related factor 2 (NRF2) response in the basal ganglia and protected against nigral dopaminergic cell death, astrogliosis, and microgliosis suggesting a neuroprotective role of sulforaphane metabolites in PD [76]. NRF2 acts as a brake in the inflammatory response by inducing antioxidant enzymes, antagonizing NF-kb, and directly inhibiting the expression of proinflammatory cytokines. NRF2 deficiency can lead to more severe dopaminergic dysfunction and can exacerbate astrogliosis and microgliosis [77].

\subsection{Ginseng reduces oxidation and inflammation}

Ginsenoside is a main ingredient in panax ginseng root. It possesses both anti-oxidative and anti-inflammatory properties. It protects against damaging oxidation by maintaining glutathione levels. Ginseng is able to regulate several inflammatory pathways including NF-kb. It has been demonstrated that ginsenoside protects against dopaminergic neuronal loss induced by toxins. Ginsenoside reduces apoptosis to reduce cell death [78].

\subsection{Vitamin E and PD risk}

The tocopherols (vitamin E) are the most important naturally occurring lipophilic antioxidants due to their ability to protect cell membranes from oxidation. Several studies have shown no effect of varying levels of vitamin E on PD risk. This may be partially because average intake of vitamin $\mathrm{E}$ is less than half of the recommended minimum amount (7 mg versus $15 \mathrm{mg}$ daily in the U.S.) [79]. Another reason that some studies do not show an effect of vitamin E on risk of PD is because they are using the less effective synthetic form of alpha-tocopherol without the other tocopherols. Interestingly, a strong protective effect against PD was found after long-term consumption of products such as peanuts, peanut butter, salad oil, or dressing, known to contain high amounts of vitamin E. Also, PD patients were found to have a reduced consumption of food rich in vitamin E compared to controls. Consumption of nuts had a strong protective association with PD [80]. Also, vitamin E reduces cyclooxygenase, lipoxygenase, and phospholipase to reduce neuroinflammation [81].

\subsection{Silymarin protects dopaminergic neurons}

Some compounds have been found to rescue dopaminergic neurons from toxic insults. The polyphenolic flavonoid silymarin is found in milk thistle (Silybum Marianum). Silymarin protects dopaminergic neurons against lipopolysaccharide-induced neurotoxicity by inhibiting microglial activation.

\subsection{S-adenosylmethionine increases glutathione}

Inflammatory TNF- $\alpha$ reduces antioxidant capacity by reducing glutathione, thus increasing injury. Sadenosylmethionine (SAM) increases glutathione and may represent a potential therapeutic intervention to protect these neurons [82].

\subsection{Polyphenols like quercetin enhance antioxidants and have an anti-inflammatory effect}

Quercetin, a flavone found in foods such as tea, grapes, onions, apples, tomatoes, and berries was found to augment electron transport chain complex I and enhance antioxidant enzyme activity [83].

\subsection{Ginkgo biloba extract is neuroprotective and reduces inflammation}

Ginkgo biloba extract is reported to have positive effects on memory. Glial proliferation in the brain was significantly attenuated by ginkgo biloba extract treatment. It also reduced expression of proinflammatory cytokines (TNF- $\alpha$, IL-1 $\beta$, and IL-6), toll-like receptor-4, and RAGE. Ginkgo biloba extract has specific neuroprotective effects and vasodilatory properties that may be useful for the treatment of cerebral hypoperfusion [84]. Ginkgo biloba extract may also exert its 
beneficial effects in patients suffering from neurological disorders through down-regulation and suppression of IL-6 secretion. After 8 weeks of treatment, patients with neurological disorders had IL-6 levels comparable with controls [85]. Ginkgo biloba extracts showed high inhibition of TNF- $\alpha$ due to NF-kb pathway impairment [86]. Ginkgo also reduced $\mathrm{C}$-reactive protein and other inflammatory and oxidative stress biomarkers after a 2-month treatment with $120 \mathrm{mg}$ of ginkgo extract twice daily [87].

\section{Conclusion}

In summary, the links between inflammation, oxidative stress, and PD have become clearer due to an overwhelming number of studies that strongly implicate inflammatory processes in the progressive loss of nigral dopaminergic neurons. Higher inflammation is associated with poor motor function and cognition in PD. Inflammation has been found even in newly-diagnosed cases. Inflammation can lead to oxidation, mitochondrial dysfunction, and death of neurons. This review is unique, to our knowledge, in that it considers dietary triggers of inflammation and non-pharmacological ways to ameliorate chronic inflammation. There are several dietary compounds that may increase risk and progression of PD that can be avoided. Other dietary compounds can be used to reduce chronic inflammation and oxidation, potentially reducing risk and progression of PD. While clinical trials have shown an association of dietary components and PD risk and progression, more randomized, controlled clinical trials are needed.

\section{Compliance with ethical standards}

\section{Acknowledgments}

I thank my fellow authors for their expert assistance.

\section{Disclosure of conflict of interest}

No Conflict of interest.

\section{References}

[1] Gundersen V. Parkinson's Disease: Can Targeting Inflammation Be an Effective Neuroprotective Strategy? Front. Neurosci. 2021; 14:580311.

[2] Marogianni C. et al.. Neurodegeneration and Inflammation-An Interesting Interplay in Parkinson's Disease. Int. J. Mol. Sci. 2020; 21: 8421.

[3] Hilker R. et al. Disease progression continues in patients with advanced Parkinson's disease and effective subthalamic nucleus stimulation. J. Neurol. Neurosurg. Psychiatry.2005; 76: 1217-1221.

[4] Burtscher J. et al. Hypoxia, Acidification and Inflammation: Partners in Crime in Parkinson's Disease Pathogenesis? Immuno. 2021; 1: 78-90.

[5] Marogianni C. et al. Neurodegeneration and Inflammation-An Interesting Interplay in Parkinson's Disease. Int. J. Mol. Sci. 2020; 21: 8421.

[6] Schonhoff A. et al. Innate and adaptive immune responses in Parkinson's disease. Prog Brain Res . 2020; 252: 169-216.

[7] Gundersen V. Parkinson's Disease: Can Targeting Inflammation Be an Effective Neuroprotective Strategy? Front. Neurosci. 2021; 14:580311.

[8] Pajares M. et al. Inflammation in Parkinson's Disease: Mechanisms and Therapeutic Implications. Cells. 2020; 9: 1687.

[9] Li S, Zhang Q, Gao Y, Nie K, Liang Y, Zhang Y, Wang L Serum Folate, Vitamin B12 Levels, and Systemic ImmuneInflammation Index Correlate With Motor Performance in Parkinson's Disease: A Cross-Sectional Study. Frontiers in Neurology. 2021; 12.

[10] Schonhoff A. et al. Innate and adaptive immune responses in Parkinson's disease. Prog Brain Res. 2020; 252: 169216.

[11] Hirsch EC. et al. Glial cells and inflammation in Parkinson's disease: a role in neurodegeneration? Ann. Neurol. 1998; 44: S115-S120. 
[12] Boka G. Hirsch EC. Immunocytochemical analysis of tumor necrosis factor and its receptors in Parkinson's disease. Neurosci. Lett. 1994; 172: 151-154.

[13] Fernandez-Checa JC. et al. GSH transport in mitochondria: defense against TNF-induced oxidative stress and alcohol-induced defect. Am. J. Physiol. 1997; 273: G7-G17.

[14] Kalampokini S. et al. 2019. Nonpharmacological Modulation of Chronic Inflammation in Parkinson's Disease: Role of Diet Interventions. Parkinson's Disease. 2019.

[15] Marogianni C. et al. Neurodegeneration and Inflammation-An Interesting Interplay in Parkinson's Disease. Int. J. Mol. Sci. 2020; 21: 8421.

[16] Pajares M. et al. Inflammation in Parkinson's Disease: Mechanisms and Therapeutic Implications. Cells 2020; 9: 1687.

[17] Minghetti L. et al. Microglial activation in chronic neurodegenerative diseases: roles of apoptotic neurons and chronic stimulation. Brain Res. Brain Res. Rev. 2005; 48: 251-256.

[18] Chen H. et al. Nonsteroidal anti-inflammatory drugs and the risk of Parkinson disease. Arch. Neurol.2003; 60: 1059-1064.

[19] Poly TN. et al. Non-steroidal anti-inflammatory drugs and risk of Parkinson's disease in the elderly population: a meta-analysis. Eur. J. Clin. Pharmacol.Jan 2019;75(1):99-108.

[20] Pajares M. et al. Inflammation in Parkinson's Disease: Mechanisms and Therapeutic Implications. Cells 2020, 9, 1687.

[21] Orr CF, Rowe DB, Halliday GM. An inflammatory review of Parkinson's disease. Prog. Neurobiol. 2002; 68: 325340 .

[22] Kim YS, Joh TH. Microglia, major player in the brain inflammation: their roles in the pathogenesis of Parkinson's disease. Exp. Mol. Med.2006; 38: 333-347.

[23] Marogianni C. et al. Neurodegeneration and Inflammation-An Interesting Interplay in Parkinson's Disease. Int. J. Mol. Sci. 2020; 21: 8421.

[24] Gundersen V. Parkinson's Disease: Can Targeting Inflammation Be an Effective Neuroprotective Strategy? Front. Neurosci.2021; 14:580311.

[25] Floor E, Wetzel MG. Increased protein oxidation in human substantia nigra pars compacta in comparison with basal ganglia and prefrontal cortex measured with an improved dinitrophenylhydrazine assay. J. Neurochem. 1998; 70: 268-275.

[26] Tilleux S, Hermans E. Neuroinflammation and regulation of glial glutamate uptake in neurological disorders. J. Neurosci. Res. 2007; 85: 2059-2070.

[27] Tansey MG. et al. Neuroinflammatory mechanisms in Parkinson's disease: Potential environmental triggers, pathways, and targets for early therapeutic intervention, Experimental Neurology. 2007; 208: 1-25.

[28] Vukajlović JM. et al. Antioxidant status and clinicopathological parameters in patients with Parkinson's disease. Vojnosanitetski pregled. 2020;77(7).

[29] Sofic E. et al. Reduced and oxidized glutathione in the substantia nigra of patients with Parkinson's disease. Neurosci. Lett. 1992; 142: 128-130.

[30] Zhang J. et al. Parkinson's disease is associated with oxidative damage to cytoplasmic DNA and RNA in substantia nigra neurons. Am. J. Pathol. 1999; 154: 1423-1429.

[31] Burtscher J. et al. Hypoxia, Acidification and Inflammation: Partners in Crime in Parkinson's Disease Pathogenesis? Immuno. 2021; 1: 78-90.

[32] Fan J, Watanabe T. Inflammatory reactions in the pathogenesis of atherosclerosis. J. Atheroscler. Thromb. 2003; 10: 63-71.

[33] Marshall KA. et al. The neuronal toxicity of sulfite plus peroxynitrite is enhanced by glutathione depletion: implications for Parkinson's disease. Free Radic. Biol. Med.1999; 27: 515-520.

[34] Tarozzi A. et al. Sulforaphane as a Potential Protective Phytochemical against Neurodegenerative Diseases. Oxidative Medicine and Cellular Longevity. 2013. 
[35] Su X. et al. Synuclein activates microglia in a model of Parkinson's disease. Neurobiol. Aging (May 28, Epub).2007.

[36] Nagatsu T. et al. Cellular and molecular mechanisms of Parkinson's disease: neurotoxins, causative genes, and inflammatory cytokines. Cell. Mol. Neurobiol.2006; 26: 781-802.

[37] Gundersen V. Parkinson's Disease: Can Targeting Inflammation Be an Effective Neuroprotective Strategy? Front. Neurosci. 2021; 14:580311.

[38] Schonhoff A. et al. Innate and adaptive immune responses in Parkinson's disease. Prog Brain Res . 2020; 252: 169-216.

[39] Betarbet R. et al. Chronic systemic pesticide exposure reproduces features of Parkinson's disease. Nat. Neurosci. 2000; 3: 1301-1306.

[40] Qu Y, Chen X, Xu MM, Sun Q. Relationship between high dietary fat intake and Parkinson's disease risk: A metaanalysis. Neural regeneration research. 2019; 14(12): 2156.

[41] Maccarrone M, Melino G, Finazzi-Agro A. Lipoxygenases and their involvement in programmed cell death. Cell Death Differ.2001; 8: 776-784.

[42] Bhattacharyya D, Bhunia A. Gut-Brain axis in Parkinson's disease etiology: The role of lipopolysaccharide. Chemistry and Physics of Lipids. 2021; 235: 105029.

[43] Wieckowska-Gacek A. et al. Western diet as a trigger of Alzheimer's disease: From metabolic syndrome and systemic inflammation to neuroinflammation and neurodegeneration. Ageing Research Reviews. 2021; 70: 101397.

[44] Harte, A. Et al. High Fat Intake Leads to Acute Postprandial Exposure to Circulating Endotoxin in Type 2 Diabetic Subjects. Diabetes Care. 2012; 35:375-382.

[45] Bailey M, Holscher H. Microbiome-Mediated Effects of the Mediterranean Diet on Inflammation. Adv Nutr. 2018;9:193-206.

[46] Ghoshal S. et al. Chylomicrons promote intestinal absorption of lipopolysaccharides. J. Lipid Res. 2009; 50: 9097.

[47] Lacroix S. et al. The bacterial endotoxin lipopolysaccharide has the ability to target the brain in upregulating its membrane CD14 receptor within specific cellular populations. Brain Pathology. 1998; 8: 625-640.

[48] Gayle DA. et al. Lipopolysaccharide (LPS)-induced dopamine cell loss in culture: roles of tumor necrosis factoralpha, interleukin-1beta, and nitric oxide. Brain Res. Dev. Brain Res.2002; 133: 27-35.

[49] Castano A. et al.. Lipopolysaccharide intranigral injection induces inflammatory reaction and damage in nigrostriatal dopaminergic system. J. Neurochem. 1998; 70: 1584-1592.

[50] White AJ, Wijeyekoon RS, Scott KM, Gunawardana NP, Hayat S, Solim IH, McMahon HT, Barker RA, Williams-Gray $\mathrm{CH}$. The peripheral inflammatory response to alpha-synuclein and endotoxin in Parkinson's disease. Frontiers in neurology.2018;9:946.

[51] Niehaus I, Lange JH. Endotoxin: is it an environmental factor in the cause of Parkinson's disease?. Occupational and environmental medicine. 2003; 60(5): 378-378.

[52] Chen H. et al. Consumption of Dairy Products and Risk of Parkinson's Disease. Am J . 2007;165:998-1006.

[53] Gaenslen, A. et al. Nutrition and the risk for Parkinson's disease: review of the literature. J Neural Transm. 2008; 115:703-713.

[54] Jiang W. et al. Dairy foods intake and risk of Parkinson's disease: a dose-response meta-analysis of prospective cohort studies. 2014. Eur J Epidemiol.

[55] Shah SP, Duda JE. Dietary modifications in Parkinson's disease: A neuroprotective intervention?. Medical hypotheses. 2015; 85(6): 1002-1005.

[56] Park M. et al. Consumption of milk and calcium in midlife and the future risk of Parkinson disease. Neurology. 2005; 005(64):1047-1051.

[57] Nedić O, Rattan SIS, Grune T, Trougakos IP. Molecular effects of advanced glycation end products on cell signalling pathways, ageing and pathophysiology. Free Radical Research,2013; 47(1): 28-38. 
[58] Derk J, MacLean M, Juranek J, Schmidt AM. The receptor for advanced glycation endproducts (RAGE) and mediation of inflammatory neurodegeneration. Journal of Alzheimer's disease \& Parkinsonism. 2018; 8(1).

[59] Udomruk S, Wudtiwai B, Shwe TH, Phitak T, Pothacharoen P, Phimphilai M,Kongtawelert P. Sesamin suppresses advanced glycation end products induced microglial reactivity using BV2 microglial cell line as a model. Brain Research Bulletin. 2021; 172:190-202.

[60] Kalampokini S. et al. Nonpharmacological Modulation of Chronic Inflammation in Parkinson's Disease: Role of Diet Interventions. Parkinson's Disease. 2019.

[61] Nilsson A. Et al. A Cereal-Based Evening Meal Rich in Indigestible Carbohydrates Increases Plasma Butyrate the Next Morning. J. Nutr.2010; 140: 1932-1936.

[62] Aho V. et al. Relationships of gut microbiota, short-chain fatty acids, inflammation, and the gut barrier in Parkinson's disease. Molecular Neurodegeneration. 2021; 16:6.

[63] Enck P. et al. A vegan or vegetarian diet substantially alters the human colonic faecal microbiota. European Journal of Clinical Nutrition. 2011; 66(1): 53-60.

[64] Kalampokini S. et al. Nonpharmacological Modulation of Chronic Inflammation in Parkinson's Disease: Role of Diet Interventions. Parkinson's Disease. 2019.

[65] Shah SP, Duda JE. Dietary modifications in Parkinson's disease: A neuroprotective intervention?. Medical hypotheses. 2015; 85(6):1002-1005.

[66] Sharma N. et al. Curcumin protects dopaminergic neurons against inflammation mediated damage and improves motor dysfunction induced by single intranigral lipopolysaccharide injection. Inflammopharmacol. 2017; 25(3): 351-368.

[67] Abbott RD. et al. Environmental, life-style, and physical precursors of clinical Parkinson's disease: recent findings from the Honolulu-Asia Aging Study. Journal of neurology. 2003; 250(3):iii30-iii39.

[68] Ungaro F. et al. Actors and Factors in the Resolution of intestinal inflammation: Lipid Mediators As a New Approach to Therapy in inflammatory Bowel Diseases. Front. Immunol. 2017; 8:1331.

[69] Kalampokini S. Nonpharmacological Modulation of Chronic Inflammation in Parkinson's Disease: Role of Diet Interventions. Parkinson's Disease. 2019.

[70] Bisht K. Et al. Curcumin, resveratrol and flavonoids as anti-inflammatory, cyto- and DNA-protective dietary compounds. Toxicology. 2010; 278: 88-100.

[71] Hang L. et al. Nutraceuticals in Parkinson's Disease. Neuromol Med. 2016; 18:306-321.

[72] Park H. et al. Dietary Antioxidants and Parkinson's Disease. Antioxidants. 2020; 9: 570.

[73] Shah SP, Duda JE. Dietary modifications in Parkinson's disease: A neuroprotective intervention?. Medical hypotheses. 2015; 85(6):1002-1005.

[74] Hang L. et al. Nutraceuticals in Parkinson's Disease. Neuromol Med. 2016; 18:306-321.

[75] Shah SP, Duda JE. Dietary modifications in Parkinson's disease: A neuroprotective intervention?. Medical hypotheses. 2015; 85(6):1002-1005.

[76] Tarozzi A. et al. Sulforaphane as a Potential Protective Phytochemical against Neurodegenerative Diseases. Oxidative Medicine and Cellular Longevity. 2013.

[77] Pajares M. et al. Inflammation in Parkinson's Disease: Mechanisms and Therapeutic Implications. Cells. 2020; 9: 1687.

[78] Hang L. et al. Nutraceuticals in Parkinson's Disease. Neuromol Med. 2016; 18:306-321.

[79] Cervantes B. et al. Vitamin E and Alzheimer's Disease-Is It Time for Personalized Medicine? Antioxidants. 2017; 6: 45 .

[80] Gaenslen A et al. Nutrition and the risk for Parkinson's disease: review of the literature. J Neural Transm. 2008; 115:703-713.

[81] Joshi Y et al. Vitamin E in aging, dementia, and Alzheimer's disease. Biofactors. March/April 2012; 38(2): 90-97.

[82] Fernandez-Checa et al. GSH transport in mitochondria: defense against TNF-induced oxidative stress and alcoholinduced defect. Am. J. Physiol.1997; 273: G7-G17. 
[83] Shah SP, Duda JE. Dietary modifications in Parkinson's disease: A neuroprotective intervention?. Medical hypotheses. 2015; 85(6): 1002-1005.

[84] Kim M et al. Ginkgo biloba L. extract protects against chronic cerebral hypoperfusion by modulating neuroinflammation and the cholinergic system. Phytomedicine. 2016; 23: 1356-1364.

[85] Ching-Hsiang L et al. In vivo effects of Ginkgo biloba extract on interleukin-6 cytokine levels in patients with neurological disorders. Indian Journal of Pharmacology. February 2012; 44: 1.

[86] Piazza S. et al. Comparison of Two Ginkgo biloba L. Extracts on Oxidative Stress and Inflammation Markers in Human Endothelial Cells. Mediators of Inflammation. 2019; 12.

[87] Siegel G et al. Combined lowering of low grade systemic inflammation and insulin resistance in metabolic syndrome patients treated with Ginkgo biloba. Atherosclerosis. 2014; 237; 584e588.

\section{Authors short Biography}

Steve Blake, ScD is a doctor of science in nutritional biochemistry. He is Director of Nutritional
Neuroscience at the Maui Memory Clinic and research director at the Neuroscience Nutrition
Foundation. He recently published a clinical study in the Journal of Brain Sciences on reversing
dementia. He has presented grand rounds at many hospitals. He authored 16 major works on the
effects of nutrition on health, including Nutrients for Memory (www.drsteveblake.com).

